\title{
Minimum MSE Equalization of Digital Fiber Optic Systems
}

\author{
DAVID G. MESSERSCHMITT, MEMBER, IEEE
}

\begin{abstract}
The optimum minimum MSE equalization, both linear and decision feedback, of a digital fiber optic transmission system with Poisson signal statistics and additive wide-sense stationary noise is considered. The problem is reduced to the solution of a certain integral equation, with solutions obtained for certain special cases, including white and band-limited additive noise. Numerical results are given for white noise and an exponential input pulse.
\end{abstract}

\subsection{INTRODUCTION}

$\mathbf{T}$ HE performance of linear equalization for a digital fiber optic system has been determined for the raised cosine family of equalized average pulses [1]. In [2] this author reported a solution for the optimum equalization with no constraint on the equalized pulses for a mean-square error criterion, including decision feedback as well as linear equalization. This paper elaborates on [2], provides the promised closed-form solution of the governing integral equation for some special cases and also some closed form solutions for the exponential pulse case. A generalization of the integral equation derived here to the $m$-ary case has been given in [8]

\subsection{MODEL OF THE RECEPTION}

At the input to the equalizer (output of the optical detector) we assume a current waveform of the form $\operatorname{er}(t)(e=$ electron charge), where

$$
r(t)=\sum_{n} \delta\left(t-t_{n}\right)+n(t)
$$

and $n(t)$ is a wide-sense stationary random process with autocorrelation $R_{n}(\tau)$ and power spectrum $N(\omega)$ and the $\left\{t_{n}\right\}$ are arrival times obeying Poisson statistics with time-varying rate parameter

$$
\lambda(t)=\lambda_{0}+\sum_{k} B_{k} p(t-k T) .
$$

In (2-2) $\lambda_{0}$ corresponds to dark current (current at the detector output in the absence of input optical energy), the $\left\{B_{k}\right\}$ are the sequence of data digits, possibly multilevel, and $p(t) \geqslant 0$ (with Fourier transform $P(\omega)$ ) is the detector average output in electrons/second corresponding to an isolated transmitted pulse. The detector input optical power is $h \nu p(t) / \eta$ where $\eta$ is the detector quantum efficiency and $h \nu$ is the energy of one

Manuscript received October 25, 1977.

The author was wth Bell Laboratories, Holmdel, NJ 07733. He is now with the Department of Electrical Engineering and Computer Sciences and the Electronics Research Laboratory, University of California, Berkeley, CA 94720. photon. For simplicity, in (2-1) we have not assumed avalanche detection, although it could easily be incorporated into the subsequent analysis. Further, we assume a sequence of uncorrelated data digits where

$$
E\left(B_{k}\right) \triangleq m_{B}
$$

(note that $m_{B}>0$ since $B_{k} \geqslant 0$ ) and

$$
E\left(B_{m} B_{n}\right)=\sigma_{B}^{2} \delta_{m, n}+m_{B}^{2}
$$

Independence of $\left\{B_{k}\right\},\left\{t_{n}\right\}$, and $n(t)$ is assumed.

\subsection{Model of the Receiver}

We constrain the receiver to be a linear filter followed by a sampler and threshold detector with (possibly) decision feedback. Specifically, let $h(\tau)$ be the impulse response of a linear filter with output $x(t)$ (the constant $e$ has been absorbed into $h$ ),

$$
x(t)=\sum_{n} h\left(t-t_{n}\right)+\int n(\tau) h(t-\tau) d \tau .
$$

To make a decision on $B_{k}$, the waveform $x(t)$ is sampled at $t=k T$ and applied to a set of thresholds. To account for the possibility of decision feedback, we also subtract prior to the thresholds the quantity

$$
\sum_{m=1}^{\infty} b_{m} \hat{B}_{k-m}+C
$$

where the $\left\{b_{m}\right\}$ are the coefficients of a feedback filter (all zero for the linear equalizer) and the $\hat{B}_{k-m}$ are past decisions. In calculating receiver performance, we will presume that there have been no past decision errors, so that $\hat{B}_{k-m}=B_{k-m}$, $m \geqslant 1$. The constant $C$ will be chosen to minimize the MSE, and is helpful because of a d.c. offset in the equalizer filter output due to dark current and other factors.

\subsection{Calculation of $M S E$}

The criterion of optimization will be the MSE between the decision threshold input and the current data digit, $B_{k}$ :

$$
\overline{e^{2}}=E\left(x(k T)-\sum_{m=1}^{\infty} b_{m} B_{k-m}-C-B_{k}\right)^{2} .
$$

In Appendix A it is shown that $(2-6)$ is minimized by

$$
C=\lambda_{0} \int h(\tau) d \tau+m_{B} \sum_{m} r_{m}-m_{B} \sum_{m=0}^{\infty} b_{m}
$$


where $b_{0} \triangleq 1$, and further that the MSE is

$$
\overline{e^{2}}=\int \psi(\tau) h^{2}(-\tau) d \tau+\overline{e_{c}^{2}}
$$

and $\overline{e_{c}{ }^{2}}$ is the MSE of the classical equalizer without shot-noise,

$$
\begin{aligned}
\overline{e_{c}{ }^{2}}= & \iint R_{n}(\tau-s) h(-\tau) h(-s) d \tau d s \\
& +\sigma_{B}{ }^{2} \sum_{m} r_{m}^{2}+\sigma_{B}^{2} \sum_{m=0}^{\infty}\left(b_{m}^{2}-2 b_{m} r_{m}\right) .
\end{aligned}
$$

In (2-7) through (2-9)

$$
r_{k}=\int p(u+k T) h(-u) d u
$$

is the average equalizer response at sample times to an isolated input pulse, and

$$
\psi(t)=\lambda_{0}+m_{B} \sum_{m} p(t-m T)
$$

is a periodic function with period $T$ and has Fourier series representation

$$
\psi(t)=\lambda_{0}+\frac{m_{B}}{T} \sum_{n} P\left(n \frac{2 \pi}{T}\right) e^{j 2 \pi n t / T} .
$$

Observe from (2-8) that for the MSE criterion the effect of the shot noise nature of the signal is to add an effective nonstationary noise with autocorrelation $\psi(t) \delta(t-\tau)$ to the input wide sense stationary noise. The subsequent solution for the optimum equalizer depends strongly on the fact that $\psi(t)$ is periodic.

We list below the four standard criteria we will use for optimization and the MSE formulas for those criteria, where the factor

$$
\begin{aligned}
F(h)= & \int \psi(u) h^{2}(-u) d u \\
& +\iint R_{n}(u-\sigma) h(-u) h(-\sigma) d u d \sigma
\end{aligned}
$$

is common to all cases:

Linear Equalizer, MSE Criterion (LE-MSE)

Minimize (2-8) with the constraint $b_{m}=0, m>0$,

$$
\overline{e^{2}}=F(h)+\sigma_{B}^{2}\left\{\left(r_{0}-1\right)^{2}+\sum_{m \neq 0} r_{m}^{2}\right\}
$$

Linear Equalizer, Zero-Forcing Criterion ( $L E-Z F)$

Minimize (2-8) with the constraint $b_{m}=0, m>0$ and $r_{k}=$ $0, k \neq 0, r_{0}=1$,

$$
\overline{e^{2}}=F(h) \text {. }
$$

Decision-Feedback Equalizer, MSE Criterion (DFE-MSE) Minimize (2-8) with no constraints,

$$
\begin{aligned}
\overline{e^{2}}= & F(h)+\sigma_{B}^{2}\left\{\sum_{m=-\infty}^{-1} r_{m}^{2}+\left(r_{0}-1\right)^{2}\right. \\
& \left.+\sum_{m=1}^{\infty}\left(b_{m}-r_{m}\right)^{2}\right\} .
\end{aligned}
$$

\section{Decision-Feedback Equalizer-Zero-Forcing Criterion} (DFE-ZF)

Minimize (2-8) with the constraint $r_{k}=0, k<0, r_{0}=1$,

$$
\overline{e^{2}}=F(h)+\sigma_{B} 2 \sum_{m=1}^{\infty}\left(b_{m}-r_{m}\right)^{2}
$$

\subsection{CONDITIONS FOR MINIMUM MSE}

The minimization of $\overline{e^{2}}$ given in (2-14)-(2-17) over $h(t)$ is straightforward by the variational method. In each case the specification of $h(t)$ is in the form of an integral equation:

$$
\begin{gathered}
\psi(t) h(-t)+\int R_{n}(t-s) h(-s) d s \\
\quad=\sigma_{B}{ }^{2} \sum_{m} C_{m} p(t+m T) .
\end{gathered}
$$

The different cases differ only as to choice of the constants $\left\{C_{m}\right\}$, which we summarize below (note that the $r_{m}$ depend on $h(t))$ :

LE-MSE

$C_{m}=\left\{\begin{array}{ll}1-r_{0}, & m=0 \\ -r_{m}, & m \neq 0\end{array}\right.$.

$L E-Z F$

The $\left\{C_{m}\right\}_{m \neq 0}$ chosen to satisfy the constraints

$r_{m}=\delta_{m}$

DFE-MSE

$C_{m}= \begin{cases}0, & m>0 \\ 1-r_{0}, & m=0 \\ -r_{m}, & m<0\end{cases}$
$b_{m}=r_{m}, \quad m>0$.

$D F E-Z F$

$C_{m}=0, \quad m>0$

$b_{m}=r_{m}, \quad m>0$. 
The $\left\{C_{m}\right\}_{m \leqslant 0}$ are chosen to satisfy the constraints

$$
r_{m}= \begin{cases}0, & m<0 \\ 1, & m=0\end{cases}
$$

In addition, it is shown in Appendix B that in all four cases

$$
\overline{e^{2}}=\sigma_{B}^{2} C_{0}
$$

\subsection{SOLUTION OF INTEGRAL EQUATION (3-1)}

An analytical solution of (3-1) can be obtained for some interesting special cases, most notably when the noise spectrum is white, periodic, or bandlimited. Most any spectrum of interest can be approximated by one of these cases. Furthermore, when $\psi(t)$ is constant, (3-1) reverts to a classical problem which is easily solved.

\subsection{General Form of the Solution of (3-1)}

It will be shown in Section 4.3 that the solution of (3-1) can be written in the form

$$
h(-t)=\sigma_{B}^{2} \sum_{m} C_{m} g(t+m T)
$$

where $g(t)$ is some time waveform which is dependent on $\lambda_{0}$, $T, m_{B}$, and $P(\omega)$, but not on $\sigma_{B}{ }^{2}$ or $\left\{C_{m}\right\}$. The conclusion of (4-1) is that the optimum equalizer consists of a filter with impulse response $g(t)$ (not necessarily a matched filter) followed by a transversal filter with tap-coefficients $\left\{C_{m}\right\}$. The latter depend on the criterion of optimization, and will be found shortly.

Our approach will be to first find the tap gains $\left\{C_{m}\right\}$ as a function of $g(t)$ in Section 4.2 and then find $g(t)$ itself in Section 4.3.

\subsection{Solution for the Tap Gains}

Section 3.0 gives relationships between the tap gains $\left\{C_{m}\right\}$ and the average equalized pulse samples $\left\{\boldsymbol{r}_{k}\right\}$. In fact, (2-10) and (4-1) give another relationship,

$$
r_{k}=\sigma_{B}^{2} \sum_{m} C_{m} S_{k-m}
$$

where

$$
S_{k}=\int p(t+k T) g(t) d t
$$

is specified by $g(t)$ and not by the tap gains. Taking the $z$ transform of (4-2), we get

$$
\begin{aligned}
R(z) & =\sum_{k} r_{k} z^{k} \\
& =\sigma_{B}{ }^{2} C(z) S(z) .
\end{aligned}
$$

Although it has not been shown yet, we assume in the following that $S(z)$ is real-valued and non-negative on the unit circle.

We now use (4-4) and the results of Section 3.0 to determine the tap gains. Once the tap gains are determined, then so is the performance through (3-9).

\section{$L E-M S E$}

From (3-2) and (4-4) we have

$$
C(z)=\frac{1}{1+\sigma_{B}{ }^{2} S(z)}
$$

and hence from (3-9)

$$
\overrightarrow{e^{2}}=\frac{T}{2 \pi} \int_{-\pi / T}^{\pi / T} \frac{\sigma_{B}{ }^{2} d \omega}{1+\sigma_{B}{ }^{2} S\left(e^{j \omega T}\right)} .
$$

\section{$L E-Z F$}

From (3-3) and (4-4)

$$
C(z)=\frac{1}{\sigma_{B}^{2} S(z)}
$$

and

$$
\overline{e^{2}}=\frac{T}{2 \pi} \int_{-\pi / T}^{\pi / T} \frac{d \omega}{S\left(e^{j \omega T}\right)}
$$

which is independent of ${\sigma_{B}}^{2}$.

\section{DFE-MSE}

From (3-4) and (4-4),

$$
\begin{aligned}
r_{k} & =\sigma_{B}{ }^{2} \sum_{m=-\infty}^{0} C_{m} S_{k-m} \\
& =\sigma_{B}{ }^{2}\left(S_{k}-\sum_{m=-\infty}^{0} r_{m} S_{k-m}\right), \quad k \leqslant 0 .
\end{aligned}
$$

The solution to this equation is well known [6]. Let

$$
\begin{aligned}
\frac{1}{\sigma_{B}^{2}}+S(z) & =S^{+}(z) S^{-}(z) \\
S^{+}(z) & =\sum_{m=0}^{\infty} \gamma_{m} z^{m} \\
S^{-}(z) & =S^{+}\left(z^{-1}\right)
\end{aligned}
$$

be the standard spectral factorization of $S(z)$. Then the solution of $(4-9)$ is

$$
\sum_{n=-\infty}^{0} r_{n} z^{n}=1-\frac{1}{\sigma_{B}{ }^{2} \gamma_{0} S^{-}(z)}
$$

and in particular

$$
r_{0}=1-\frac{1}{{\sigma_{B}}^{2} \gamma_{0}^{2}}
$$


where

$$
\gamma_{0}^{2}=\exp \left\{\frac{T}{2 \pi} \int_{-\pi / T}^{\pi / T} \ln \left[S\left(e^{j \omega T}\right)+\frac{1}{\sigma_{B}^{2}}\right] d \omega\right\} .
$$

Hence, from (3-9) we have

$$
\overline{e^{2}}=\gamma_{0}^{-2}
$$

\section{$D F E-Z F$}

From (3-6) (3-8), and (4-4),

$$
r_{k}=\delta_{k}=\sigma_{B}^{2} \sum_{m=-\infty}^{0} C_{m} S_{k-m}, \quad k \leqslant 0 .
$$

The solution of this equation follows from [6, Appendix A, Eq. (72)] with $R(\theta)=1 / \sigma_{B}^{2}$ as

$$
\sum_{k=-\infty}^{0} C_{k} z^{k}=\frac{1}{\sigma_{B}^{2} \gamma_{0} S-(z)}
$$

where now

$$
\begin{aligned}
S(z) & =S^{+}(z) S^{-}(z) \\
\gamma_{0}{ }^{2} & =\exp \left\{\frac{T}{2 \pi} \int_{\pi / T}^{\pi / T} \ln S(\omega) d \omega\right\}
\end{aligned}
$$

and (4-16) holds as before.

\subsection{Solution for $g(t)$}

That (4-1) is a solution of (3-1) can be verified directly by substitution, with the result that $g(t)$ must satisfy the integral equation

$$
\psi(t) g(t)+\int R_{n}(t-s) g(s) d s=p(t)
$$

In Sections 4.3.1 and 4.3.2 (4-21) will be solved directly for two special cases, and then in subsequent Sections more difficult cases will be solved using the Laurent-Cauchy Transform (LCT).

\subsection{1 $\psi(t)=\psi_{0}, A$ Constant}

From (2-12) whenever $p(t)$ has no energy at the sympol rate $1 / T \mathrm{~Hz}$ or harmonics thereof, $\psi(t)$ is a constant $\psi_{0}=$ $\lambda_{0}+m_{B} P(0) / T$. This case, or an approximation to it, will frequently occur in practice. By taking the Fourier Transform of $(4-21)$ we get

$$
G(\omega)=\frac{P(\omega)}{\psi_{0}+N(\omega)}
$$

and it is easily shown that

$$
S\left(e^{j \omega T}\right)=\frac{1}{T} \sum_{n} \frac{\left|P\left(\omega+n \frac{2 \pi}{T}\right)\right|^{2}}{\psi_{0}+N\left(\omega+n \frac{2 \pi}{T}\right)}
$$

which is the classical matched filter plus whitening filter solution with the noise spectrum augmented by $\psi_{0}$.

$$
\begin{aligned}
& \text { 4.3.2 } N(\omega)=N_{0}, \text { White Noise } \\
& \text { When } N(\omega)=N_{0}, g(t) \text { becomes }
\end{aligned}
$$

$$
g(t)=\frac{p(t)}{N_{0}+\psi(t)} .
$$

In particular, when the $p(t+m T)$ pulses do not overlap, we get

$$
g(t)=\frac{p(t)}{N_{0}+\lambda_{0}+m_{B} p(t)}
$$

In either case, $g(t)$ differs from the classical solution in that it is not a matched filter, but rather has an impulse response weighted during each symbol interval according to periodic function $\psi(t)$. Interestingly, the bandwidth of the equalizer generally exceeds that of $p(t)$ when the latter is bandlimited.

Many equivalent formulas for $S(z)$ can be given, but perhaps the most useful is to substitute (4-24) in to (4-3).

\subsubsection{Transformation of (4-21) into a Difference Equation}

For more difficult cases (neither $\psi(t)$ nor $N(\omega)$ constant) it is useful to transform (4-21) into a difference equation using the concept of the Laurent-Cauchy Transform (LCT) [5]. Given a function $f(t)$ with Fourier transform $F(\omega)$, define

$$
F_{n}(\omega)=F\left(\omega+n \frac{2 \pi}{T}\right), \quad|\omega| \leqslant \frac{\pi}{T}
$$

Then the LCT of $f(t), F_{L C}(z, \omega)$, is defined as

$$
F_{\mathrm{LC}}(z, \omega)=\sum_{n} z^{n} F_{n}(\omega), \quad|\omega| \leqslant \frac{1}{T} .
$$

The inverse is easily determined by taking the inverse $z$-transform followed by the inverse Fourier transform.

Now (4-21) can be transformed into a difference equation by substituting for $\psi(t)$ from (2-12) and taking the Fourier Transform to obtain

$$
\begin{aligned}
& \left(\lambda_{0}+N(\omega)\right) G(\omega)+\frac{m_{B}}{T} \sum_{n} P_{n}(0) G\left(\omega-n \frac{2 \pi}{T}\right) \\
& \quad=P(\omega) .
\end{aligned}
$$

When $\omega$ is replaced by $\omega+m(2 \pi / T)$ and $\omega$ is restricted to 
$|\omega| \leqslant \pi / T$, we obtain

$$
\begin{aligned}
& \left(\lambda_{0}+N_{m}(\omega)\right) G_{m}(\omega)+\frac{m_{B}}{T} \sum_{n} P_{n}(0) G_{m-n}(\omega) \\
& =P_{m}(\omega), \quad|\omega| \leqslant \pi / T
\end{aligned}
$$

which is a linear difference equation with time-varying coefficients for fixed $\omega$.

The solution to (4-28) is facilitated by taking the $z$-transform of both sides,

$$
\begin{gathered}
\left(\lambda_{0}+\frac{m_{B}}{T} P_{\mathrm{LC}}(z, 0)\right) G_{\mathrm{LC}}(z, \omega) . \\
+\sum_{m=-\infty}^{\infty} N_{m}(\omega) G_{m}(\omega) z^{m} \\
=P_{\mathrm{LC}}(z, \omega), \quad|\omega| \leqslant \pi / T
\end{gathered}
$$

There are two special cases, considered in the following two sections, for which (4-29) can be solved.

\subsection{4 $N(\omega)$ Periodic in $N 2 \pi / T$}

A case for which (4-29) can be solved occurs when

$$
N\left(\omega+N \frac{2 \pi}{T}\right)=N(\omega)
$$

or equivalently

$$
N_{k+N}(\omega)=N_{k}(\omega) \text {. }
$$

When $N=1$, the solution follows immediately as

$$
\begin{gathered}
G_{\mathrm{LC}}(z, \omega)=\frac{P_{\mathrm{LC}}(z, \omega)}{\lambda_{0}+\frac{m_{B}}{T} P_{\mathrm{LC}}(z, 0)+N(\omega)}, \\
|\omega| \leqslant \pi / T .
\end{gathered}
$$

The similarity to (4-22) is striking. For $N>1$, solution is still possible, but tedious. For example, when $N=2$, we can write $P_{\mathrm{LC}}(z, \omega)$ and $G_{\mathrm{LC}}(z, \omega)$ each as the sum of two terms, one containing even powers of $z$ and the other odd powers of $z$,

$$
\begin{aligned}
& P_{\mathrm{LC}}(z, \omega)=P_{e}(z, \omega)+P_{0}(z, \omega) \\
& G_{\mathrm{LC}}(z, \omega)=G_{e}(z, \omega)+G_{0}(z, \omega)
\end{aligned}
$$

and substituting into (4-31) we get two equations, one for even powers,

$$
\begin{gathered}
{\left[\lambda_{0}+\frac{m_{B}}{T} P_{e}(z, 0)+N_{0}(\omega)\right] G_{e}(z, \omega)} \\
\quad+\frac{m_{B}}{T} P_{0}(z, 0) G_{0}(z, \omega) \\
=P_{e}(z, \omega)
\end{gathered}
$$

and the other for odd powers,

$$
\begin{aligned}
& \frac{m_{B}}{T} P_{0}(z, 0) G_{e}(z, \omega) \\
& \quad+\left[\lambda_{0}+\frac{m_{B}}{T} P_{e}(z, 0)+N_{1}(\omega)\right] G_{0}(z, \omega) \\
& =P_{0}(z, \omega) .
\end{aligned}
$$

These two equations can be solved simultaneously for $G_{e}(z, \omega)$ and $G_{0}(z, \omega)$ from which $G_{\mathrm{LC}}(z, \omega)$ can be constructed.

An alternate method of solution for $N>1$ is to rewrite (4-28) as a vector difference equation with constant coefficients, for which solution is straightforward [7]. For example, when $N=2$ and $P_{n}(0)=0,|n|>1,(4-28)$ can be rewritten as

$$
\begin{aligned}
X_{m+1}= & A_{m} X_{m}+Y_{m} \\
X_{m}= & {\left[G_{m}(\omega) G_{m-1}(\omega)\right]^{T} } \\
A_{m}= & \frac{1}{P_{-1}(0)} \\
& \cdot\left[\begin{array}{ccc}
-\frac{m_{B}}{T}\left(\lambda_{0}+N_{m}(\omega)\right)-P_{\mathbf{0}}(0) & -P_{1}(0) \\
1 & 0
\end{array}\right] \\
Y_{m}= & \frac{T}{m_{B} P_{-1}(0)}\left[\begin{array}{lll}
P_{m}(\omega) & 0
\end{array}\right]^{T} .
\end{aligned}
$$

Using the fact that $A_{2 k}=A_{0}$ and $A_{2 k+1}=A_{1},(4-37)$ can be rewritten in the form of another first order difference equation with constant coefficients,

$$
X_{2 k+2}=A_{1} A_{0} X_{2 k}+A_{1} Y_{2 k}+Y_{2 k+1} .
$$

\subsubsection{N( $\omega)$ Bandlimited}

If we assume that $N(\omega)=0$ for $|\omega| \geqslant(N+1 / 2)(2 \pi / T)$ then it follows that $N_{m}(\omega)=0$ for $|m|>N$ and $|\omega| \leqslant \pi / T$. Accordingly, (4-29) becomes

$$
\begin{gathered}
{\left[\lambda_{0}+\frac{m_{B}}{T} P_{\mathrm{LC}}(z, 0)\right] G_{\mathrm{LC}}(z, \omega)} \\
\quad+\sum_{m=-N}^{N} N_{m}(\omega) G_{m}(\omega) z^{m} \\
=P_{\mathrm{LC}}(z, \omega), \quad|\omega| \leqslant \pi / T .
\end{gathered}
$$

This equation can be solved by writing $G_{k}(\omega)$ in terms of $G_{\mathrm{LC}}(z, \omega)$,

$$
G_{k}(\omega)=\frac{1}{T} \int_{0}^{T} e^{-j 2 \pi k t / T} G_{\mathrm{LC}}\left(e^{j 2 \pi t / T}, \omega\right) d t
$$


and substituting for $G_{\mathrm{LC}}$ from (4-39) to obtain

$$
G_{k}(\omega)=a_{k}(\omega)-\sum_{m=-N}^{N} b_{m-k} N_{m}(\omega) G_{m}(\omega)
$$

where

$$
\begin{aligned}
a_{k}(\omega) & =\frac{1}{T} \int_{0}^{T} e^{-j 2 \pi k t / T} \psi^{-1}(t) P_{\mathrm{LC}}\left(e^{j 2 \pi t / T}, \omega\right) d t \\
b_{k} & =\frac{1}{T} \int_{0}^{T} e^{-j 2 \pi k t / T} \psi^{-1}(t) d t
\end{aligned}
$$

For $|k| \leqslant N,(4-41)$ is a set of $(2 N+1)$ equations in $(2 N+1)$ unknowns which can be solved by matrix inversion. For $|k|>$ $N G_{k}(\omega)$ can then be determined directly from (4-41).

\subsection{EXAMPLE}

In [2] a numerical example is given for a Gaussian shaped optical power pulse, which is characteristic of relatively long optical fiber links with significant mode conversion [8]. Here we will concentrate on an exponential input pulse [1], primarily because its analytical tractability is conducive to gaining significant insight. The receiver input pulse is

$$
p(t)= \begin{cases}\alpha n_{e} e^{-\alpha t}, & t>0 \\ 0, & t<0\end{cases}
$$

where $n_{e}$ is the average number of detector output electrons per bit, and the rms pulse width is $\sigma_{0}=\alpha^{-1}$. For convenience we define

$$
\beta=e^{-\alpha t}=e^{-1 / \sigma}
$$

where $\sigma=\sigma_{0} / T$ is the rms pulse width in time slots. By straightforward calculation,

$$
\psi(t)=\lambda_{0}+\frac{m_{B} n_{e} \alpha}{1-\beta} e^{-\alpha T}, \quad 0 \leqslant t \leqslant T
$$

Consider the white noise case of Section 4.3.2. An important parameter is

$$
\rho=\frac{T\left(\lambda_{0}+N_{0}\right)}{n_{e}}
$$

which is the ratio of average number of noise electrons per time slot to signal electrons per transmitted symbol (with $\left.B_{k}=1\right)$. Then $g(t)$ is given by (4-23),

$$
g(t+k T)= \begin{cases}\frac{\beta_{k}}{\sigma \rho e^{\alpha t}+m_{B} /(1-\beta)}, & 0 \leqslant t \leqslant T \\ 0, & t \geqslant 0\end{cases}
$$
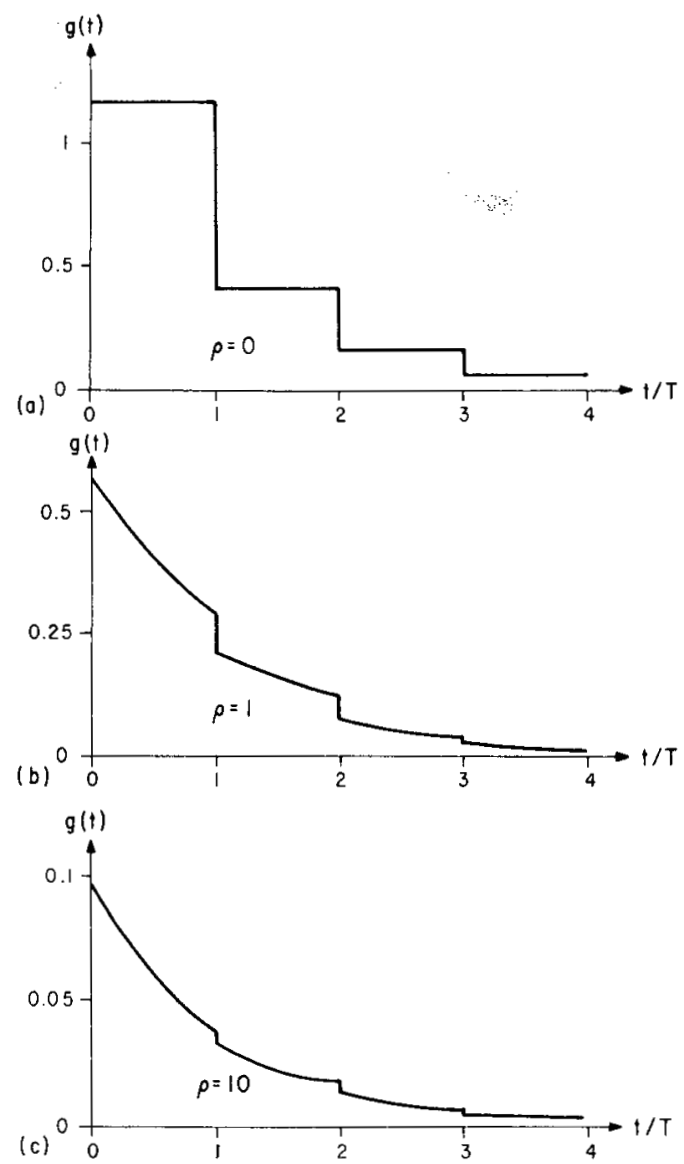

Figure 1. Impulse Response of Optimum Equalizer Prefilter.

This impulse response is plotted in Fig. 1 for $m_{B}=0.5$ and $\sigma=1$ as $\rho$ varies. When $\rho=0, g(t)$ is interestingly a sum of square pulses, and as $\rho$ increases, $g(t)$ approaches the shape of the input pulse (i.e., a classical matched filter for white noise).

Where $p(t)$ is causal and $g(t)$ is given by (4-23), $S_{k}$ in (4-3) can be written in the form

$$
S_{k}=\sum_{m=l}^{\infty} \int_{0}^{T} \frac{p(u+(m+k) T) p(u+m T)}{N_{0}+\psi(u)} d u
$$

where $l=-k$ for $k<0$ and $l=0$ for $k \geqslant 0$. Substituting from (5-1), after some manipulation, we get

$$
S_{k}=\frac{K}{1-\beta^{2}} \beta^{|k|}
$$

and hence

$$
S(z)=\frac{K}{(1-\beta z)(1-\beta / z)}
$$

where

$$
K=\frac{n_{e}(1-\beta)^{2}}{m_{B}{ }^{2}}\left[m_{B}-\sigma \rho \log _{e}\left(\frac{\sigma \rho(1-\beta)+m_{B}}{\sigma \rho(1-\beta)+m_{B} \beta}\right)\right] .
$$


The tap-gains are readily determined from (5-8) and from

$$
\begin{aligned}
\frac{1}{\sigma_{B}{ }^{2}}+S(z) & =\frac{\beta}{a \sigma_{B}{ }^{2}} \frac{(1-a z)(1-a / z)}{(1-\beta z)(1-\beta / z)} \\
a & =b-\sqrt{b^{2}-1}<1 \\
b & =\frac{1+\sigma_{B}^{2} K+\beta^{2}}{2 \beta} .
\end{aligned}
$$

The tap-gains and MSE of all four equalizers are now readily determined (the latter using (3-9)):

LE-MSE

$$
\begin{gathered}
C(z)=\frac{a(1-\beta z)(1-\beta / z)}{\beta(1-a z)(1-a / z)} \\
\overline{e^{2}}=\frac{a \sigma_{B}^{2}}{\beta}\left\{1+\frac{(a-\beta)^{2}}{1-a^{2}}\right\} .
\end{gathered}
$$

$L E-Z F$

$$
\begin{aligned}
C(z) & =\frac{(1-\beta z)(1-\beta / z)}{K \sigma_{B}{ }^{2}} \\
\overline{e^{2}} & =\frac{1+\beta^{2}}{K} .
\end{aligned}
$$

\section{DFE-MSE}

$$
\begin{aligned}
S^{+}(z) & =\sqrt{\frac{\beta}{a \sigma_{B}{ }^{2}}} \frac{1-a z}{1-\beta z} \\
\overline{e^{2}} & =\frac{a \sigma_{B}{ }^{2}}{\beta} .
\end{aligned}
$$

$D F E-Z F$

$$
\begin{aligned}
S^{+}(z) & =\frac{\sqrt{K}}{1-\beta z} \\
\overline{e^{2}} & =\frac{1}{K} .
\end{aligned}
$$

The reduction in MSE for the DFE relative to the LE is evident from these equations for both the MSE and ZF criteria.

As pointed out in [2], the most meaningful way to present an example is to plot the increase in input optical power required to maintain a constant MSE (and hopefully relatively constant error rate). This is done in Fig. 2 for the same example as in Fig. 1. Comparing with the Gaussian pulse example in [2], we see that the penalties are somewhat smaller for the exponential pulse (intersymbol interference is less severe for a given $\sigma$ ). Further, the performance advantage in going to the more complicated receiver structure of the DFE is not nearly as large for the same reason. For longer multi-mode fibers with

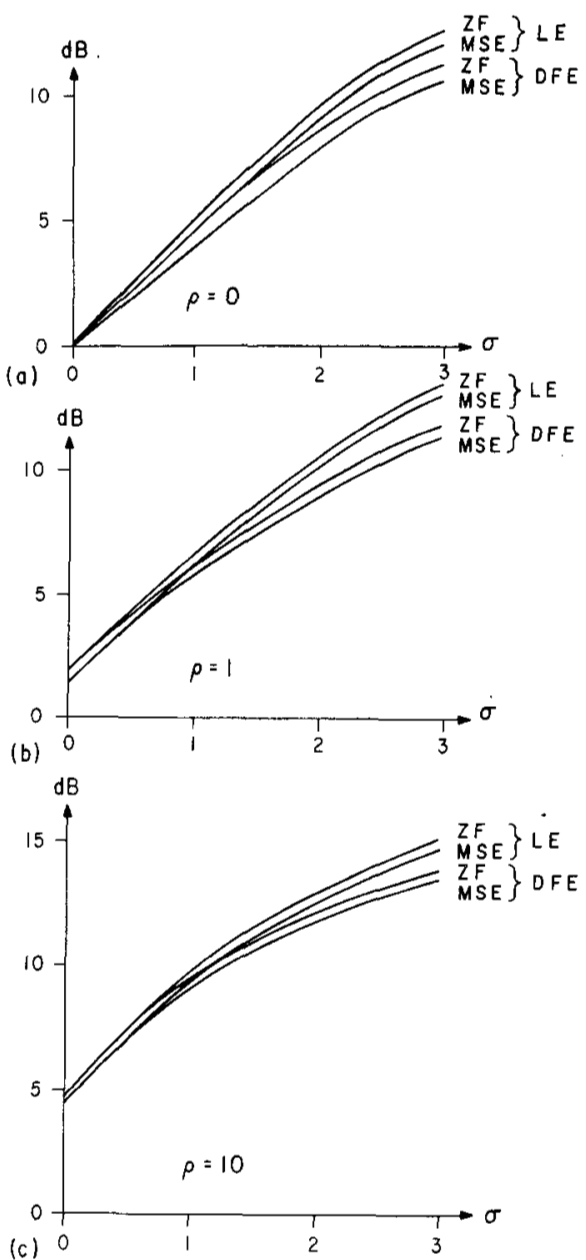

Figure 2. Penalty in Receiver Optical Power (Relative to $\sigma=0$, $\rho=0)$ Required to Maintain SNR $=16.28(\mathrm{~dB})$.

significant mode conversion, the Gaussian model of [2] is more germaine.

\subsection{CONCLUSIONS}

The most interesting aspect of the solution of the MMSE equalization problem is the emergence of a transversal filter prceeded by a nonmatched filter as the solution.

The most serious shortcomings of the present work are twofold: First, the restriction of consideration to linear equalization or decision-feedback equalization, while possibly conforming to practical constraints, does not give insight into the benefit of arbitrarily complex processing. Second, the MSE criterion, while being extremely tractable, is not as desirable a criterion of optimization as would be the error rate. Both of these shortcomings are addressed with some success in [9-10] .

\section{APPENDIX A}

Defining

$$
\begin{aligned}
& A=x(k T)-K \\
& K=\sum_{m=0}^{\infty} b_{m} B_{k-m}, \quad b_{0}=1
\end{aligned}
$$


we get

$$
\overline{e^{2}}=E(A-C)^{2} \text {. }
$$

Minimizing with respect to $c$, we get

$$
\begin{aligned}
c & =E(A) \\
\overline{e^{2}} & =E\left(A^{2}\right)-E(A)^{2} .
\end{aligned}
$$

We take these expectations first over the Poisson statistics, using Campbells Theorem [1],

$$
\begin{aligned}
& E\left(\sum_{n} h\left(k T-t_{n}\right)\right)^{2} \\
& \quad=\int \lambda(\tau) h^{2}(k T-\tau) d \tau+\left(\int \lambda(\tau) h(k T-\tau) d \tau\right)^{2} \\
& E\left(\sum_{n} h\left(k T-t_{n}\right)\right) \\
& \quad=\int \lambda(\tau) h(k T-\tau) d \tau
\end{aligned}
$$

and then over the data. First, since $n(t)$ has zero mean, and using (A.6)

$$
\begin{aligned}
& E\left(x^{2}(k T)\right) \\
& =\lambda_{0} \int h^{2}(\tau) d \tau+\sum_{n} B_{n} \int p(\tau-n T) h^{2}(k T-\tau) d \tau \\
& \quad+\lambda_{0}^{2}\left(\int h(\tau) d \tau\right)^{2}+2 \lambda_{0} \int h(\tau) d \tau \sum_{m} B_{m} r_{k-m} \\
& \quad+\sum_{m} \sum_{n} B_{m} B_{n} r_{k-m} r_{k-n}+\iint R_{n}(\tau-s) h(-\tau) \\
& \quad \cdot h(-s) d \tau d s .
\end{aligned}
$$

Similarly,

$$
E(x(k T))=\lambda_{0} \int h(\tau) d \tau+\sum_{m} B_{m} r_{k-m}
$$

Now we are prepared to take the expectation over the data utilizing (2-3) and (2-4). First, we have that

$$
\begin{aligned}
& E(x(k T))=\lambda_{0} \int h(\tau) d \tau+m_{B} \sum_{m} r_{m} \\
& E(K)=m_{B} \sum_{m=0}^{\infty} b_{m}
\end{aligned}
$$

$E\left(x^{2}(k T)\right)$

$$
\begin{aligned}
& =\lambda_{0} \int h^{2}(\tau) d \tau+m_{B} \sum_{n} \int p(\tau-n T) h^{2}(k T-\tau) d \tau \\
& \quad+\lambda_{0}\left(\int h(\tau) d \tau\right)^{2}+2 \lambda_{0} m_{B} \int h(\tau) d \tau \sum_{m} r_{m} \\
& \quad+m_{B}^{2} \sum_{m} \sum_{n} r_{k-m_{m}} r_{k-n}+\sigma_{B}^{2} \sum_{m} r_{m}^{2} \\
& \quad+\iint R_{n}(\tau-s) h(-\tau) h(-s) d \tau d s
\end{aligned}
$$

$E(x(k T) K)$

$$
\begin{gathered}
=\lambda_{0} m_{B} \int h(\tau) d \tau \sum_{n=0}^{\infty} b_{n}+m_{B} 2 \sum_{m} r_{m} \sum_{n=0}^{\infty} b_{n} \\
\quad+\sigma_{B} 2 \sum_{n=0}^{\infty} r_{n} b_{n} \\
E\left(K^{2}\right)=m_{B} 2\left(\sum_{m=0}^{\infty} b_{m}\right)^{2}+\sigma_{B} 2 \sum_{m=0}^{\infty} b_{m}^{2} .
\end{gathered}
$$

Finally, these terms can be combined in (A.5) to obtain

$$
\begin{aligned}
\overline{e^{2}}=\int \psi(\tau) h^{2}(-\tau) d \tau+\iint R_{n}(\tau-s) h(-\tau) h(-s) d \tau d s \\
+\sigma_{B}^{2}\left(\sum_{m} r_{m}^{2}+\sum_{m=0}^{\infty} b_{m}^{2}-2 \sum_{m=0}^{\infty} r_{m} b_{m}\right) .
\end{aligned}
$$

\section{APPENDIX B}

To calculate the MSE, multiply both sides of (3-1) by $h(-t)$ and integrate to obtain

$$
F(h)=\sigma_{B}^{2} \sum_{m} C_{m} r_{m}
$$

For the LE-MSE, substitute (3-2) in (B.1) to obtain

$$
F(h)=\sigma_{B}^{2}\left\{\left(1-C_{0}\right) C_{0}-\sum_{m \neq 0} C_{m}^{2}\right\}
$$

and substituting (B.2) and (3-2) in (2-14), (3-9) follows. The other cases follow by the same method.

\section{REFERENCES}

1. S. D. Personick, "Receiver Design for Digital Fiber Optic Communication System," BSTJ, Vol. 52, July-August, 1973.

2. D. G. Messerschmitt, "Optimum Mean-Square Equalization for Digital Fiber Optic Systems," Proc. Int. Conf. Communications, San Francisco, June, 1975.

3. A. Papoulis, Probability, Random Variables, and Stochastic Processes, McGraw-Hill, 1965. 
4. F. B. Hildebrand, Methods of Applied Mathematics, PrenticeHall, 1965

5. Y. H. Ku and A. A. Wolf, "Laurent-Cauchy Transform for Analysis of Linear Systems Described by Differential-Difference and Sum Equations," Proc. IRE, Vol. 48, pp. 923-931, May, 1960.

6. J. Salz, "Optimum Mean-Square Decision Feedback Equalization," BSTJ, Vol. 52, pp. 1341-1374, October, 1973.

7. E. I. Jury, Theory and Applications of Z-Transform Method, John Wiley, New York, 1964.

8. W. A. Gardner, "An Equivalent Linear Model for Marked and Filtered Doubly Stochastic Poisson Processes with Application to MMSE Linear Estimation for Synchronous $m$-ary Optical Data Signals," IEEE Trans. on Comm., Vol. COM-24, p. 917, August 1976.
9. S. D. Personick, P. Balaban, J. H. Bobsin, and P. R. Kumar, "A Detailed Comparison of Four Approaches to the Calculation of the Sensitivity of Optical Fiber System Parameters," IEEE Trans. on Comm., Vol. COM-25, p. 541, May, 1977.

10. G. J. Foschini, R. D. Gitlin, J. Salz, "Optimum Direct Detection for Digital Fiber Optic Communication Systems," BSTJ, Vol. 54, p. 1389 , October, 1975.

David G. Messerschmitt (S'65-M'68), for a photograph and biography see page 675 of the May 1978 issue of this TRANSACTIONS. 